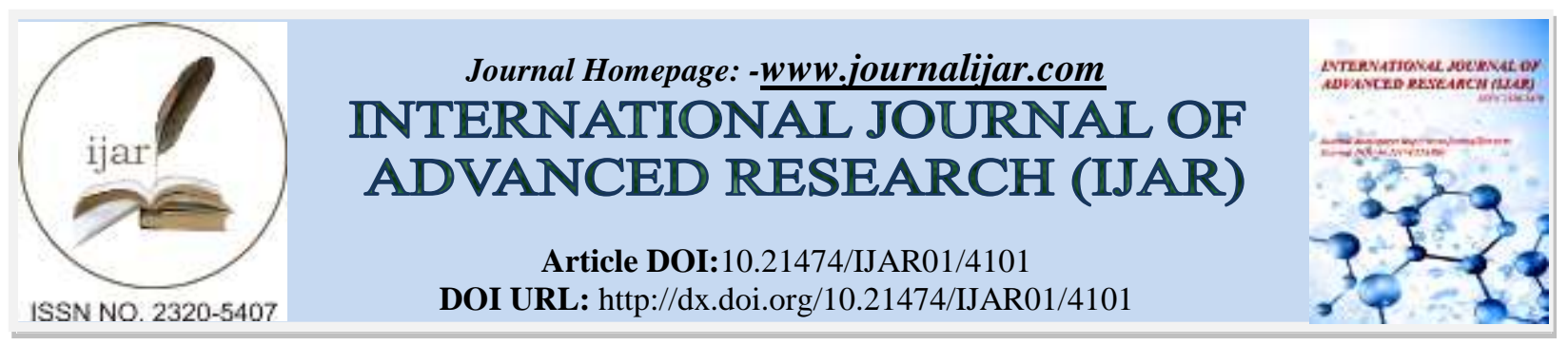

RESEARCH ARTICLE

\title{
RADIOLOGICAL IMAGING IN A CASE OF ARTERIAL TORTUOSITY SYNDROME.
}

\author{
Dr Jyoti Gupta ${ }^{1}$, Dr. Bhanu Pratap Singh Chauhan ${ }^{2}$ and Dr Zalak Panchal ${ }^{3}$. \\ 1. Resident doctor, Department of Radio diagnosis, Civil hospital, Ahmedabad. \\ 2. Resident doctor, Department of Surgery, V.S hospital,Ahmedabad. \\ 3. Resident doctor, Department of Radio diagnosis, Civil hospital, Ahmedabad
}

\section{Manuscript Info}

Abstract

Manuscript History

Received: 07 March 2017

Final Accepted: 01 April 2017

Published: May 2017

Copy Right, IJAR, 2016,. All rights reserved.

\section{Introduction:-}

A 5 year old male child presented with complaints of easy fatigability, pallor, improper growth, easy bruisability and skin hemorrhages at few sites. He was referred to our hospital for the same. On clinical examination, the patient had micrognathia, enlarged head with frontal bossing, elongated face with extreme pallor. Lax skin with hypermobility of joints was seen. Routine blood investigations were done which showed anemia (HB- 8.8 gm/dl), mild leukocytosis (TLC- 14000) and low grade fever. Abdomen was tender on palpation Chest X-ray revealed mild cardiomegaly which was also present on ECG. ECG also showed abnormal vessels and few collaterals. CT aortography was advised which was done.

\section{Imaging Findings:-}

CT angiography was done on 128 multislice CT machine. It revealed presence of elongated and tortuous vessel in thorax and abdomen. Aorta was extremely aneurysmally dilated. Thoracic and abdominal aorta showed tortuous and lax coarse. All major cerebral and abdominal vessels were also tortuous. Few collaterals vessels were also in mediastinum near origin of major cerebral vessels.

No abnormal venous channels were seen. No e/o concurrent hemorrhage seen.

Associated mild hepatosplenomegaly seen in abdomen with small defect in anteromedial right sided diaphragm with Morgagni hernia with bowel loops as its content.

Rest of the abdominal organs were normal. 


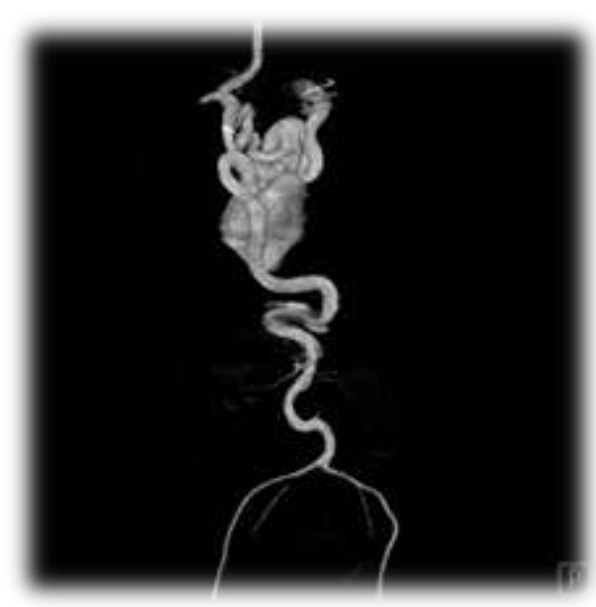

3D view of abdominal aorta and its branches

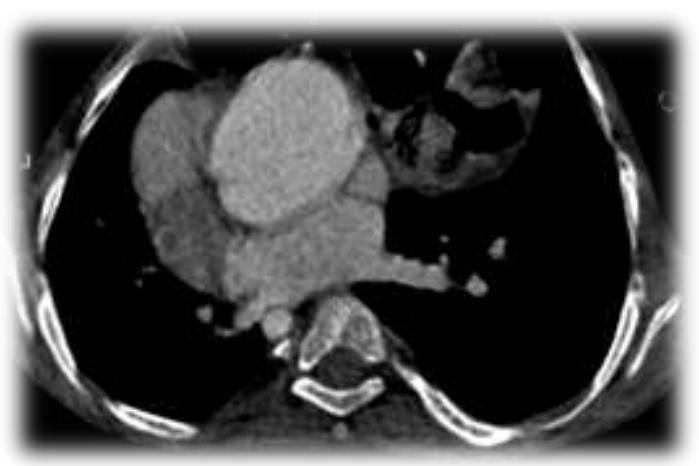

Cross section view of dilated ascending aorta

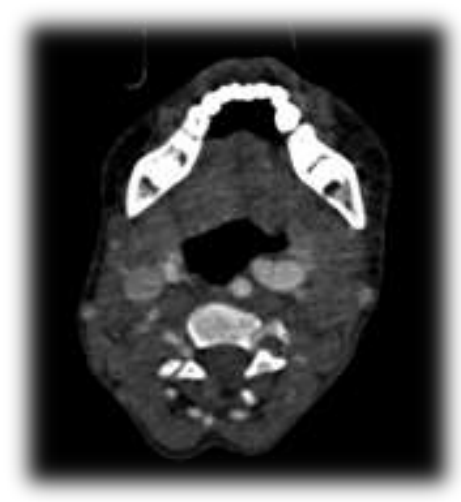

Dilated carotid arteries on either side

\section{Discussion:-}

Arterial tortuosity syndrome is a very rare congenital disorder with autosomal recessive inheritance. It primarily affects collagen with defect in SLC2A10 gene. Many mutations have been detected till date in the gene but this is the most common association. Its exact incidence and prevalence is unknown. It affect both sexes equally and presents in childhood depending upon the severity of involvement of various systems and presence of complications. It is clinical diagnosis based on abnormal facies and abnormal skin changes. Main Clinical features of the disease are dysmorphic facies - periorbital fullness, micrognathia hyperteleorism, enlarged head, high palate, dental abnormalities, delayed and retarted growth, abnormal skin aka cutis laxa. Hypermobility of joints may be seen with end stage contractures or deformities. Progressive scliotic deformties, vertebral deformities, pectus excavates or carinatum may develop.

Imaging findings reveal presence of elongated and winding tortuous major vessels. Presence of focal dilatations , narrowing and stenosis is common. Role of radiological imaging is to ascertain the diagnosis and detct complications. It helps surgeon in accurate preoperative mapping of the disease.

Additional genitourinary or gastric abnormalities such as diverticulitis , malrotations etc. may be present.

Other connective tissue findings are joint involvements, prolapse of urinary bladder, uterus ets.

Complication in the form of dissection, hemorrhage, pulmonary complications, CNS strokes due to hemorrhages or vascular constrictions may be present.

Regular clinical and radiological follow up of the patients is advised to avoid life threatening complications. It should include Electrocardiogram, CT plain and contrast with arterial phase from head to toe, MRI Brain with angiography sequences and indicated investigations as per clinical scenario. 
This disease is managed by adequate synergy between clinician, radiologist and cardiothoracic surgeon.

Differential Diagnosis:-

Autosomal recessive cutis laxa syndrome Type 1 and Loeys-dietz syndrome are major differentials. Many other common connective tissue disorders should be considered in clinical settings such as Marfan's Syndrome, Ehler Danlos Syndrome which have specific clinical features.

\section{References:-}

1. Gayathri Satish, Sheela Nampoothiri, Mahesh K Gayathri Satish, Sheela Nampoothiri, Mahesh Kappanayilappanayil, Arterial tortuosity syndrome phenotypic features and cardiovascular manifestations. circulation. ahajournals 2008;117:e477-e478

2. Erfan Mehrabi1,Kamran Khan2, Shahab A Malik1, Arterial tortuosity syndrome. BMJ case reports 2016 1136/bcr-2016-217029

3. Alkuwari M, Kamal RY, Shelby S, Maliyekkal ST, Kutty S. Low-dose CT angiography for evaluation of great vessels and airway in arterial tortuosity syndrome. Eur Heart J Cardiovasc Imaging. 2012;13:1054.[PubMed]

4. Zalak Patel et al. Rare case of Arterial Tortuosity Syndrome Preoperative and Post Operative Imaging Findings ISJR August 2016 Vol5 Issue 8 